\title{
Electron Transfer Rates in DNA Films as a Function of Tether Length
}

\author{
T. Gregory Drummond, Michael G. Hill, and Jacqueline K. Barton \\ Division of Chemistry and Chemical Engineering \\ California Institute of Technology \\ Pasadena, California 91125
}

\section{Supporting Information}

Table S-1. Tabulated electrochemical data for the series of tether lengths investigated. Figure S-1. Variation of cathodic and anodic peak potentials as a function of scan rate (in $\mathrm{V} / \mathrm{s}$ ) for the series of tethers investigated. Black squares: $n=4$; red circles: $n=5$; blue up triangles: $n=6$; dark yellow down triangles: $n=7$; purple diamonds: $n=8$; green left triangles: $n=9$. Laviron formalism was used to generate fit lines for each data set using an $\alpha$ value of 0.6. Background-subtracted cyclic voltammograms were generated by numerically interpolating the charging currents to points just before and after the onset of the respective cathodic and anodic Faradaic responses. The resulting backgrounds were then subtracted from the raw data to yield traces like those shown in Figure 2 of the text. We note that the "trumpet plots" shown above are based on uncorrected voltammograms; use of background-subtracted data instead gave peak splittings (and resulting $\mathrm{k}_{\mathrm{s}}$ values) within experimental error of those shown.

Figure S-2. Representative cyclic voltammograms for two DM-DNA films ( $n=5$ top, and $n=9$, bottom) recorded in $5 \mathrm{mM}$ phosphate, $50 \mathrm{mM} \mathrm{NaCl}, \mathrm{pH} 7.5$ at a series of scan rates. As the scan rate increases, the peak potentials shift slowly for the shorter tether but much more quickly for the longer tether due to the distance-dependence of charge transfer kinetics across the $\sigma$-bonded tether.

Figure S-3. Demonstration of background subtraction to yield corrected cyclic voltammograms. Analysis of raw and background-subtracted data yielded very similar values for $\Delta \mathrm{E}_{\mathrm{p}}$ and $\mathrm{k}_{\mathrm{s}}$.

Figure S-4. Potential dependence of the DNA film height, as measured by atomic force microscopy (AFM) under electrochemical control (for comparison, the open-circuit height is $45 \AA$ ). The inset shows a plot of the maximum film height (measured at $\sim 100$ $\mathrm{mV}$ vs. the quasi-reference electrode) measured for duplexes possessing 15, 18, and 20 bases. These data show a slope of $3.2 \AA / \mathrm{bp}$, close to the predicted value of $3.4 \AA / \mathrm{bp}$. The intercept, $\sim 7 \AA$, is somewhat smaller than the $\sim 16 \AA$ expected for a fully extended alkylthiol linker. See reference 18 a for experimental details. 
Table S-1.

\begin{tabular}{cccccc}
$\begin{array}{c}\text { \# Methylenes } \\
(\boldsymbol{n})^{\mathbf{a}}\end{array}$ & $\begin{array}{c}\mathbf{E}^{\mathbf{0}} \\
(\mathbf{m V})^{\mathbf{b}}\end{array}$ & $\begin{array}{c}\mathbf{E}_{\mathbf{p c}} \\
(\mathbf{m V})^{\mathbf{c}}\end{array}$ & $\begin{array}{c}\mathbf{E}_{\mathbf{p a}} \\
(\mathbf{m V V})^{\mathbf{d}}\end{array}$ & $\begin{array}{c}\mathbf{k}_{\mathbf{s}} \\
\left(\mathbf{s}^{\mathbf{- 1}}\right)^{\mathbf{e}}\end{array}$ & $\begin{array}{c}\Gamma_{\text {DNA-DM }} \\
\left(\mathbf{p m o l} / \mathbf{c m}^{\mathbf{2}} \mathbf{f}^{\mathbf{f}}\right.\end{array}$ \\
\hline \hline 4 & $-600( \pm 6)$ & $-605( \pm 5)$ & $-596( \pm 5)$ & $733( \pm 45)$ & $39( \pm 5)$ \\
5 & $-598( \pm 6)$ & $-603( \pm 5)$ & $-593( \pm 5)$ & $176( \pm 30)$ & $37( \pm 6)$ \\
6 & $-607( \pm 6)$ & $-613( \pm 5)$ & $-601( \pm 5)$ & $89( \pm 14)$ & $38( \pm 6)$ \\
7 & $-605( \pm 6)$ & $-612( \pm 5)$ & $-599( \pm 5)$ & $32( \pm 8)$ & $36( \pm 7)$ \\
8 & $-606( \pm 7)$ & $-613( \pm 5)$ & $-599( \pm 5)$ & $12( \pm 4)$ & $33( \pm 8)$ \\
9 & $-607( \pm 7)$ & $-616( \pm 5)$ & $-598( \pm 5)$ & $4.4( \pm 1)$ & $33( \pm 8)$ \\
\hline Averages & $-604( \pm 7)$ & $-610( \pm 6)$ & $-598( \pm 5)$ & & $36( \pm 9)$
\end{tabular}

a) Number of methylene units in $\sigma$-bonded alkydiamine portion of tether. b) Apparent formal potential of daunomycin in the DNA-modified electrode. c) Cathodic peak potentials from cyclic voltammograms at low scan rates; all potentials stated versus saturated $\mathrm{AgCl} / \mathrm{Ag}$ reference. d) Anodic peak potentials from cyclic voltammetry. e) Aparent rates of electron transfer in DNA-DM films determined by Laviron method using the value $\alpha=0.6$, which gave good fits to the experimental data. f) Surface excess of DNA-DM conjugate in monolayer, measured routinely by adsorption charge and phosphate binding assay. ${ }^{15}$ 
Figure S-1.

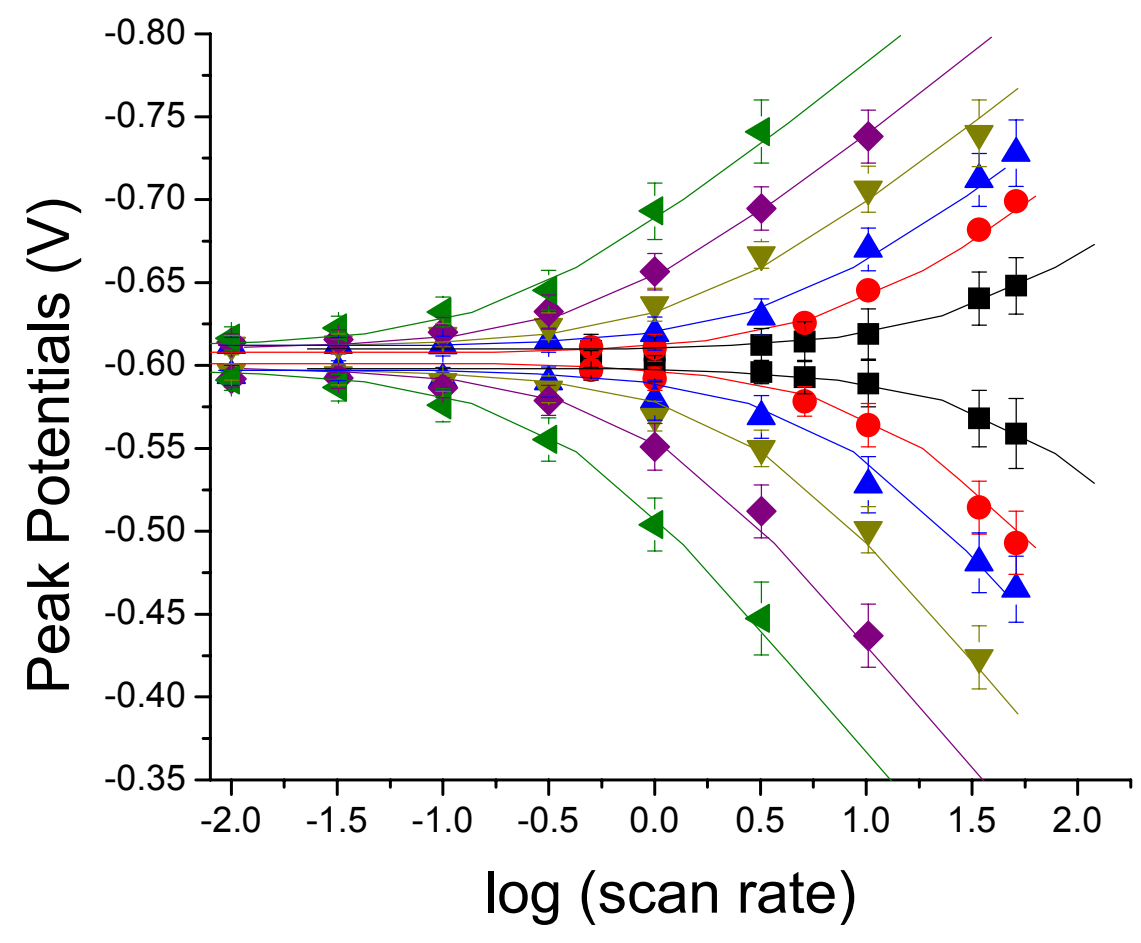


Figure S-2.
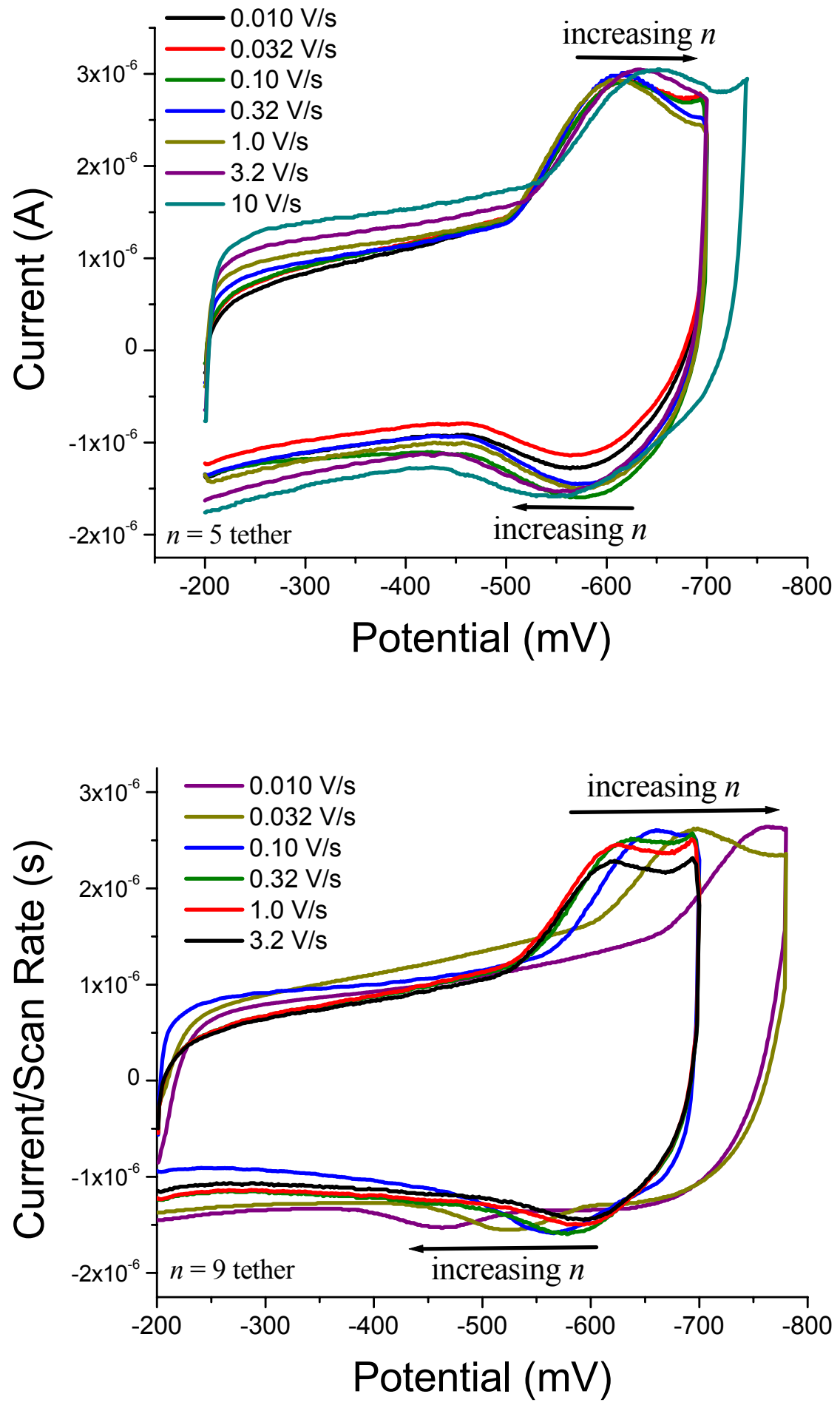
Figure S-3.

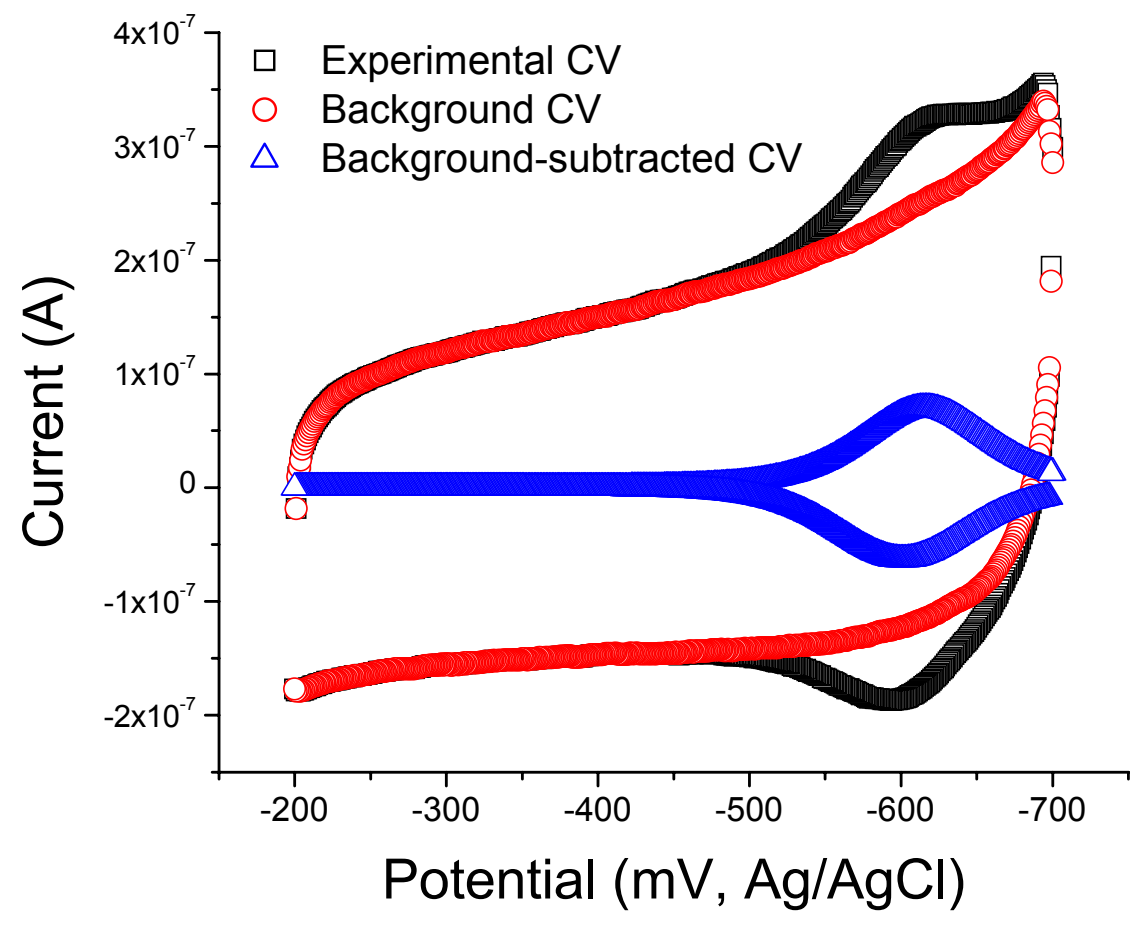


Figure S-4.

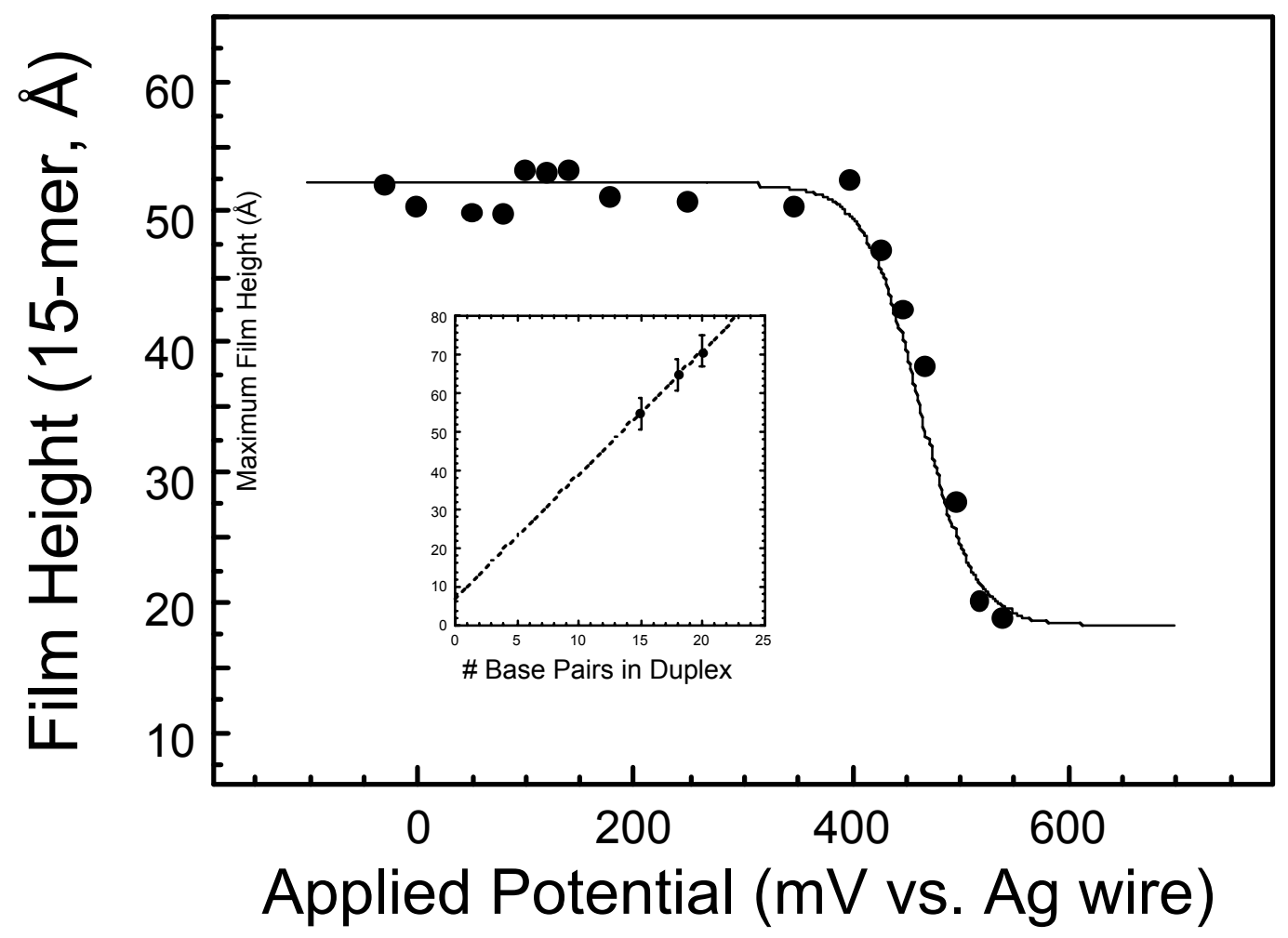

